\title{
Towards higher densities of matter: ultra-high pre-compression in shock dynamic experiments
}

\author{
A. Dwivedi ${ }^{1}$, S. Petitgirard ${ }^{2}$, K. Appel ${ }^{1}$, E. Brambrink ${ }^{1}$, Z. Konôpková ${ }^{1}$, M. Millot ${ }^{3}$, T. Preston ${ }^{1}$, A. Ravasio ${ }^{4}$, \\ C. Strohm ${ }^{5}$, U. Zastrau ${ }^{1}$, V. Cerantola ${ }^{1}$ \\ ${ }^{1}$ European X-ray Free Electron Laser facility GmbH, 22869 Schenefeld, Germany, \\ ${ }^{2}$ Department of Earth Sciences, ETH-Zürich, 8092 Zürich, Switzerland, \\ ${ }^{3}$ Lawrence Livermore National Laboratory, 94550 Livermore, USA, \\ ${ }^{4}$ Laboratoire pour l'Utilisation des Lasers Intenses, École Polytechnique, 91128 Palaiseau cedex, France, \\ ${ }^{5}$ Deutsches Elektronen-Synchrotron DESY, 22607 Hamburg, Germany

\section{dwivedi@xfel.eu}

The extreme densities of matter relevant to most exoplanets are not reachable by static compression, i.e., in diamond anvil cell (DAC), or by single shock compression techniques. Multiple shocks generated by tailored laser pulses allow higher densities to be reached, but the thermodynamic state of the system is not easy to measure. Instead of using the multi-shock compression technique, we can send a laser-induced shock wave through a sample that is pre-compressed at high static pressures inside a DAC. The equation-of-state of the system can then be directly measured through the Rankine-Hugoniot equations from the shock and particle velocities, and temperature can be measured independently with pyrometry. Several experiments demonstrated the combination of these two techniques [1-5] at $\mathrm{kJ}$ laser facilities and documented material properties at unprecedented conditions.

We introduce a new design of a shock diamond anvil cell (SDAC) for sub-kJ laser-driven dynamic compression experiments at X-ray sources. We designed a system of two thin diamond anvils, one of which is perforated. The perforation is envisioned to allow shock waves created by low/moderate energy lasers to propagate through the sample. Being developed to be usable by any user community at the High Energy Density (HED) instrument at European-XFEL, or other large-scale facilities around the world, the unique design of the SDAC will make it possible to reach higher density states of matter in dynamic compression experiments and probe previously unexplored regions of the pressure-temperature-density phase diagram, combined with x-ray techniques at XFEL sources. We will present technical details and first results of the pre-compression pressures achieved using SDAC along with hydrodynamic simulation results of dense Krypton, among other samples, laser-shocked at different initial densities.

1. Loubeyre, P. et al (2003). High Pressure Research. 24, 1, 25-31

2. Eggert, J. et al (2008). Phys. Rev. Lett. 100, 124503

3. Celliers, P. M. et al (2010). Phys. Rev. Lett. 104, 184503

4. Loubeyre, P. et al (2012). Phys. Rev. B. 86, 144115

5. Millot, M. et al (2018). Nat. Phys. 14, 297-302

Keywords: pre-compression; shock-compression; noble gases

Part of this work was prepared by LLNL under Contract DE-AC52-07NA27344 and supported by LDRD 19-ERD-031. 